P042 DOES SLEEP INERTIA AFFECT HOW WE PERCEIVE TIME? - IMPLICATIONS FOR INSOMNIA

${ }^{1}$ Megan Crawford, ${ }^{2}$ Annie Vallieres, ${ }^{3}$ Matt Salanitro, ${ }^{3}$ Hannah Rees*, ${ }^{3}$ Michelle Carr, ${ }^{3}$ Ceri Bradshaw, ${ }^{2}$ David Laroch, ${ }^{2}$ Patricia Nolin, ${ }^{2}$ Julia Delage, ${ }^{3}$ Mark Blagrove. ${ }^{1}$ University of Strathclyde, Glasgow, UK; ${ }^{2}$ Laval University, Quebec, Canada; ${ }^{3}$ Swansea University, Swansea, UK

\subsection{6/bmjresp-2019-bssconf.42}

Introduction Sleep inertia (SI) can negatively affect cognitive functions including time perception. Accurate time perception is important for evaluating wakefulness at night. Overestimating wakefulness can be anxiety-provoking for individuals with insomnia. Here we present data from three studies testing the impact of SI on time perception after waking from sleep versus after a wake period.

Methods Participants were required to complete a time estimation task after waking from sleep and after a wakeful period. In study 1 and $2(n=18)$, sleep occurred as part of a daytime nap with polysomnography. In study $3(n=9)$ sleep occurred at night in a laboratory. Study 1 and 3 included good sleepers and study 2 included poor sleepers. The time estimation task was the same across all studies asking participants to state when they believed 15 minutes had passed, see figure 1.

Results Data from study 1 and 2 were pooled. Participants overestimated time, however there was a greater overestimation after waking from a nap compared to the wake condition, $\mathrm{t}(17)=-2.089, \mathrm{p}=0.052, \mathrm{~d}=0.7$. For those individuals who reached stage 3 sleep during the nap (when waking with SI is more likely) the difference was significant, $t(9)=-3.22$, $\mathrm{p}<0.05, \mathrm{~d}=1.1)$. There was no main effect of sleep status (poor sleeper vs. good sleeper) on these differences. In study 3 there was no difference $(p>0.05)$ between the two conditions (wake vs. sleep), see figure 2 for a summary of the findings.

Conclusion Overestimation of time awake was more pronounced after waking from a nap condition compared to after a wake period, especially when waking from stage 3 sleep. The same effects were not present when waking from sleep at night, perhaps due to different study designs. Further research in larger samples is needed to understand the impact of SI on time perception.

\section{P043 PREVALENCE OF A MENTAL HEALTH DIAGNOSIS IN PATIENTS PRESCRIBED CPAP THERAPY AND ITS IMPACT ON TREATMENT ADHERENCE}

Marta Vilaca, Ana Gaspar, Claire Wotton, Jack Ridler, Priya Nair*, Joel Patasin, Alison Mcmillan. East and North Hertfordshire NHS Trust, Stevenage

\subsection{6/bmjresp-2019-bssconf.43}

Introduction Obstructive Sleep Apnoea (OSA) is a common condition, highly prevalent but under-recognized in patients with a Mental Health Diagnosis (MHD). Patients with MHD have a higher prevalence of risk factors such as obesity, diabetes, hypertension, hyperlipidaemia, tobacco smoking, alcohol consumption and sedative medication use. Continuous Positive Airway Pressure (CPAP) therapy is effective in reducing sleepiness and improving quality of life. However, treatment adherence may be low, which can compromise its effectiveness.

Method We performed a Service Audit by reviewing clinical data regarding all patients in the Sleep Unit at Lister Hospital who have been prescribed CPAP therapy between June 2011 and July $2018 \quad(n=2642)$, with the aim of determining the prevalence of MHD and characterizing the population and adherence to treatment.

Results Out of the 2642 patients who were included in the audit:

- $2018(76.4 \%)$ were using CPAP

- $624(23.6 \%)$ stopped CPAP treatment for various reasons

- $442(16.7 \%)$ had a MHD
If the participant thinks 15 minutes has passed, but it is really shorter, then they have overestimated time.

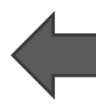

Abstract P042 Figure 1 Conceptual outline of time estimation task
If the participant thinks 15 minutes has passed, but it is really longer, then they have underestimated time.
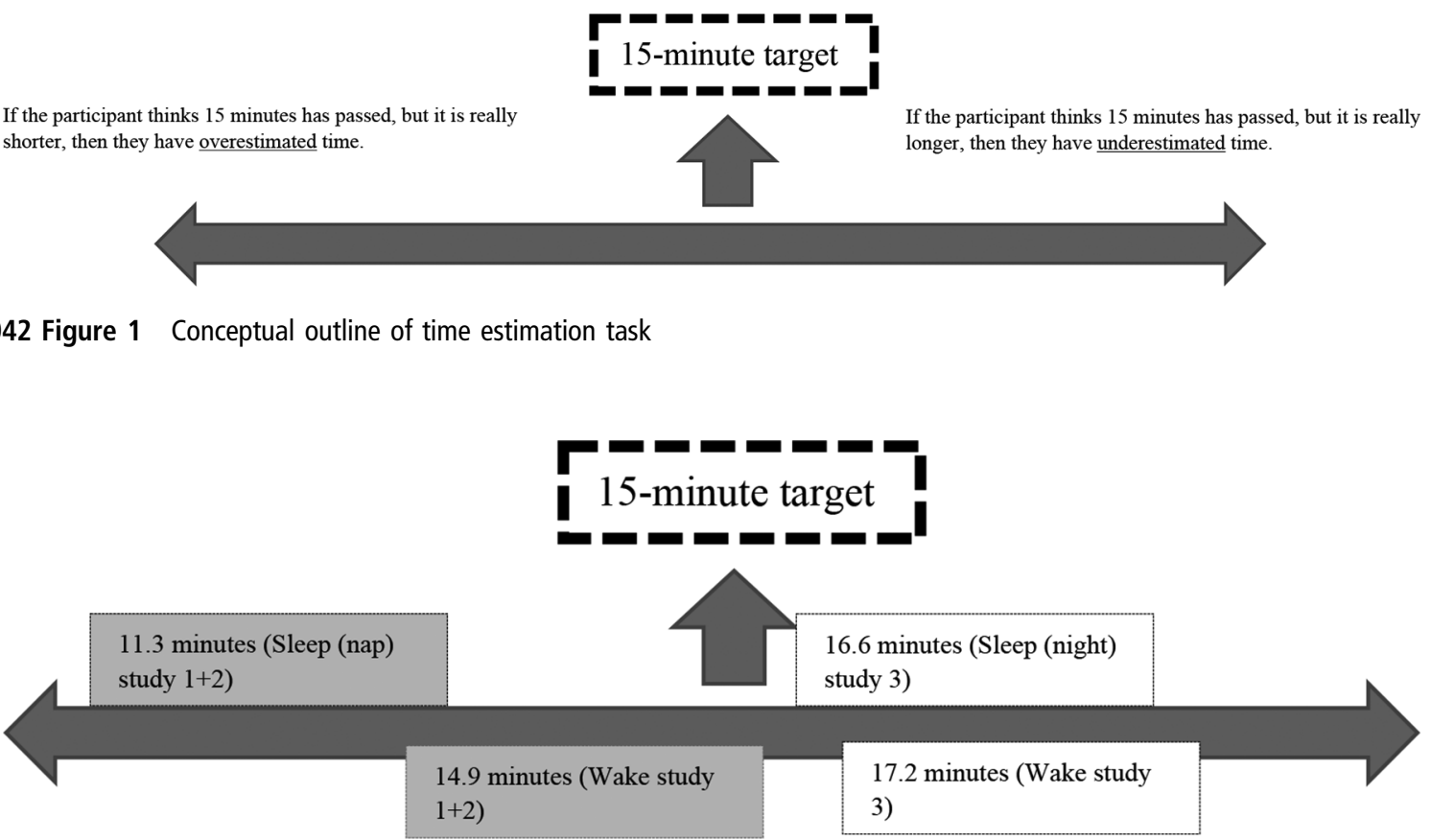

Abstract P042 Figure 2 Results of all three studies 
Abstract P043 Table 1 Baseline characteristics; data are mean $\left(25^{\text {th }}, 75^{\text {th }}\right.$ percentiles $)$

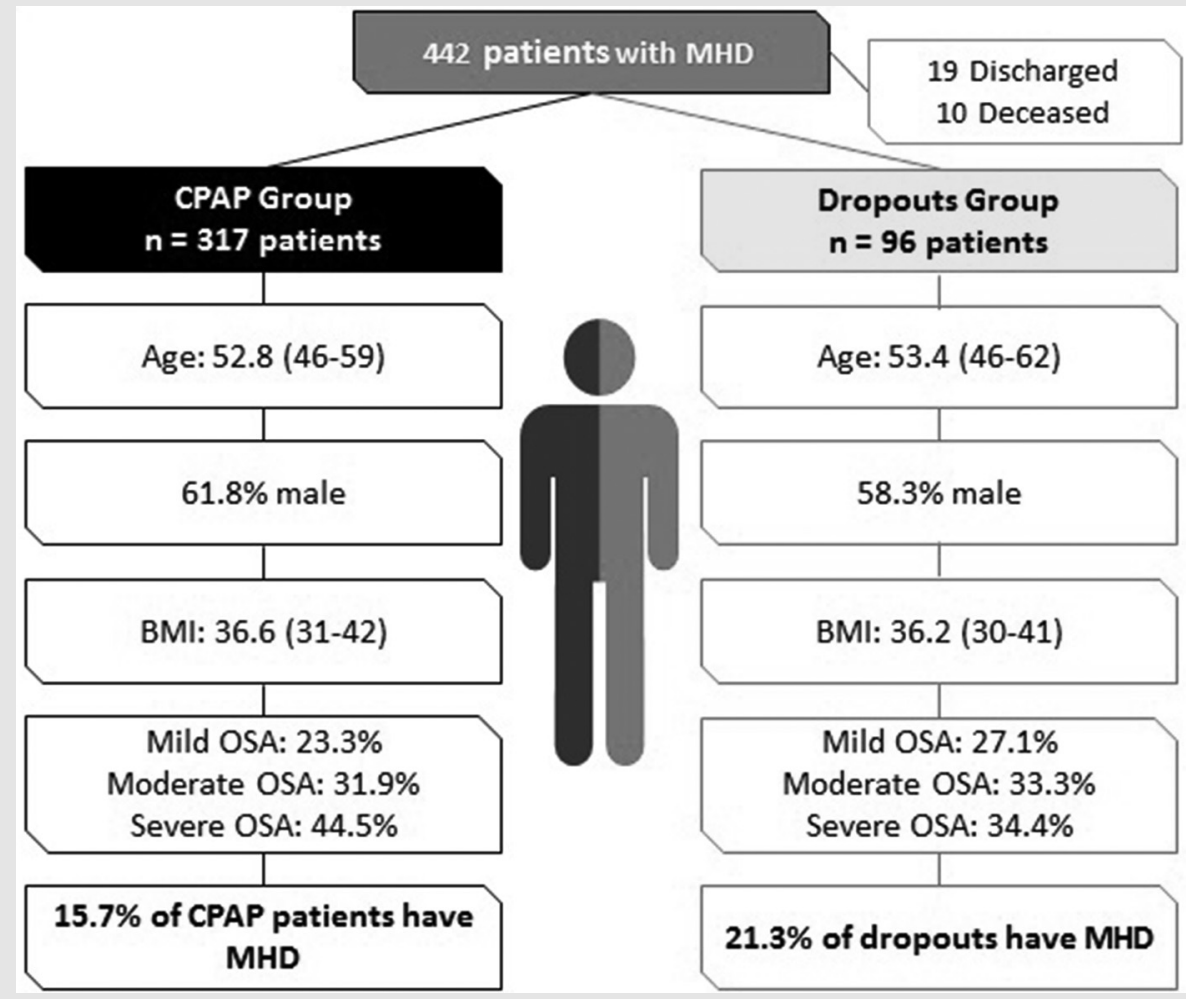

Within the MHD group, there was similar age, gender distribution and BMI. [Table 1].

Discussion The CPAP treatment adherence rate in our service is $76.4 \%$, which is higher than reported in literature. The percentage of patients with MHD is lower than expected $(16.7 \%)$, as it is estimated that $39 \%$ of the adults in the UK suffer with common mental health disorders. There is a higher percentage of $\mathrm{MHD}$ in the dropout group $(21.29 \%)$ than in the CPAP group (15.71\%), despite similar age, BMI and gender distribution, suggesting this may be a factor in achieving compliance with CPAP treatment.

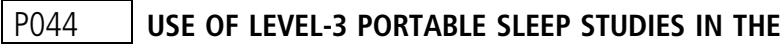 PAEDIATRIC POPULATION. USER FEEDBACK}

Sakina Dastagir*, Rishi Pabary, Ross Langley, Ridma Jayarathna, Hannah Williams. Royal Brompton, London, UK

\subsection{6/bmiresp-2019-bssconf.44}

Introduction Ambulatory sleep, cardio-respiratory devices, or level 3 sleep studies have been used for screening and diagnosis of sleep apnoea in the adult and paediatric population. The advantages of using a level 3 portable sleep monitoring device include being able to expedite diagnosis, ease for the patient and family members and being 50\% the cost of an in-patient level 1 PSG. Literature is limited on the use of these devices in paediatrics, this includes patient/guardian usability and satisfaction as well as the ability to assess sleep disorders.

From May 2018 we produced and distributed a questionnaire to address these issues.
Methods The questionnaire comprised of 4 questions about the device and the sleep study process. There are 3-6 options for each answer (see table 1). We also recorded technical failures using the report generated by the DOMINO LightTM Software auto-analyser. This includes failure rate of the pulse oximeter, respiratory bands and nasal cannulae.

Results At the time of analysis, 30 completed questionnaires demonstrated that the majority (93\%) would prefer a home study compared to an in-patient study. At the time of analysis, 31 completed questionnaire revealed that $48 \%$ of guardians found the most challenging part of the home set up was the nasal cannulae. Figure 1.

Discussion The preference for home studies suggests this method of monitoring and diagnosing patients could be beneficial for the future of our service. This is in keeping with the desire to deliver modern healthcare within the home setting so called 'hospital at home'.

The nasal cannulae provides important information for analysis and diagnosis of the study. For further analysis, the

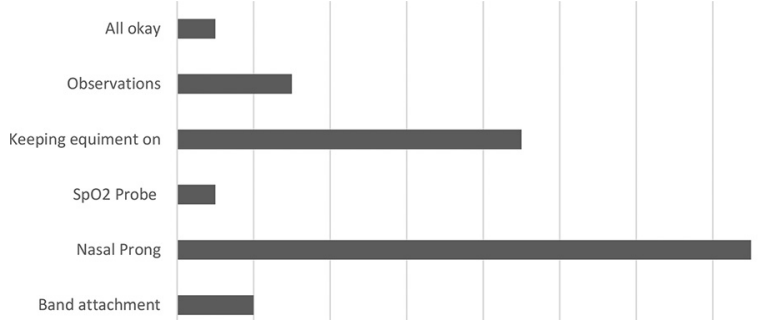

Abstract P044 Figure 1 Graph showing guardian response to the most challenging Equipment to set up 